\title{
A study of polarisation modes in PSR $0823+26$
}

\author{
Joanna M. Rankin \\ University of Vermont, Burlington, VT-05405, USA
}

\section{N. Rathnasree}

Raman Research Institute, Bangalore, 560080, INDIA

\section{Introduction}

We explore the detailed polarisation behaviour of pulsar $0823+26$ using the technique of constructing partial "mode-separated" profiles corresponding to the primary and secondary polarisation modes. The characteristics of the two polarisation modes in this pulsar are particularly interesting, both because they are anything but orthogonal (Backer \& Rankin 1980), and because the secondary mode exhibits structure seen neither in the primary mode nor in the total profile.

\section{Polarisation mode separation}

Using the model-angle method of polarisation-mode deconstruction (Rankin et. al. 1989; Cordes et. al. 1978), we can take the following representation of the single-vector model to define the respective primary and secondary-mode PA traverses

$$
\chi=\chi_{0}+\tan ^{-1} \frac{\sin \left(\phi-\phi_{0}\right)}{A-\left(A-\frac{1}{R}\right) \cos \left(\phi-\phi_{0}\right)},
$$

where $\chi$ is the PA, $\phi$ the longitude, $R=\left|\frac{d \chi}{d \phi}\right|_{\max }$ the maximum PA sweep rate, and $A$ a constant which can be computed from the total PA traverse, $\chi_{c}$ between reference longitudes $\pm \phi_{c}$ as

$$
A=\frac{\sin \phi_{c} / \tan \frac{1}{2} \chi_{c}-\cos \phi_{c} /|R|}{1-\cos \phi_{c}}
$$

and which is formally equal to $\sin \zeta / \tan \alpha$, where $\alpha$ and $\zeta$ are the angles that the magnetic axis and the sight line make with the rotation axis, respectively.

We proceed to construct partial ("mode-separated") profiles corresponding to the primary and secondary modes of $0823+26$ using necessary information for the above equation from the PA angle histograms (not shown). The details are discussed in Rankin \& Rathnasree (1996). The first, "primary-mode" partial profile, consists of those samples which had PAs lying on that half of the Poincare sphere closest to the Stokes vector representing the model PA, the second those in the other hemisphere.

The primary-mode partial profile (Fig. 1) greatly resembles the total profile (not shown). The PA traverse, now more nearly conforms to what is expected 

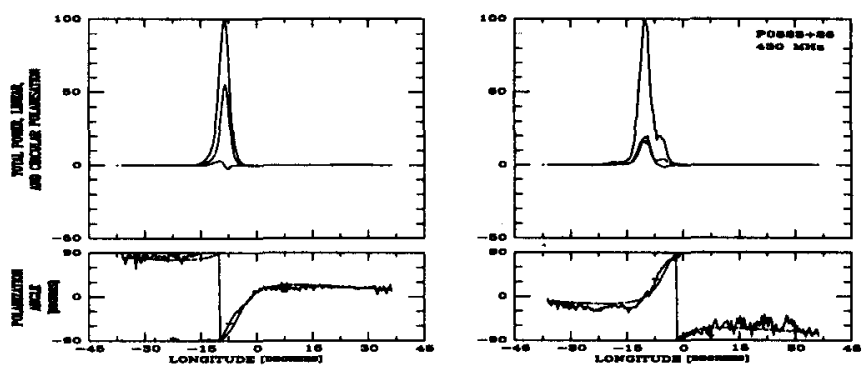

Figure 1. Partial mode separated profiles for PSR $0823+26$ at 430 MHz: primary mode (left) and secondary (right). The top panels show: total intensity (solid line), linear polarisation (dashes) and the circular power (dots). Bottom panels show position angle variations.

on the basis of the single-vector model. The postcursor (PC) is most prominent in the primary mode, and its linear polarisation appears more complete. The secondary mode profile shows a more complex structure, both in $\mathrm{L}$ and total power. Were it a complete profile, we would immediately say it had a triple form. The polarisation is low, but not insignificant, throughout its full extent.

An interesting feature of the mode-separated partial profiles is a phase offset between the primary and secondary modes. The secondary-mode "main pulse" arrives some $1.5 \pm 0.1^{\circ}$ before the primary-mode one at $430 \mathrm{MHz}$ and some $1.3 \pm 0.1^{\circ}$ at $21 \mathrm{~cm}$. Given that the polar cap has an angular diameter of $3.36^{\circ}$, we consider whether this is a geometric effect or an effect of differential propagation of the two modes in the inner magnetosphere of the pulsar.

\section{Conclusions}

We conclude that $0823+26$ has a very nearly equatorial geometry, in that both magnetic poles and the sightline all fall close to the rotational equator of the star. We thus associate the postcursor component with emission along those bundles of field lines which are also equatorial and which continue to have a tangent in the direction of our sight line for a significant portion of the star's rotation cycle. Perhaps it is emission along this bundle-at a height of some $4 \times 10^{3} \mathrm{~km}$ or about $4 \%$ of the light cylinder that the PC emission occurs. It seems that in all pulsars with postcursor components, this emission follows the core component, and all may thus have equatorial emission geometries.

Acknowledgments. This work supported in part by the US NSF (AST 89-17722). Arecibo Obs. is operated by Cornell Uni. under contract to NSF.

\section{References}

Backer, D. C., \& Rankin, J. M. 1980, ApJS, 42, 143

Cordes, J. M., Rankin, J. M., \& Backer, D. C. 1978, ApJ, 223, 961

Rankin, J. M., Campbell, D. B., \& Backer, D. C. 1974, ApJ, 188, 609

Rankin, J.M. \& Rathnasree, N. 1996, JAA, in press 